\title{
MEASURABLE LINEAR FUNCTIONALS AND OPERATORS ON FRÉCHET SPACES
}

\author{
ANDRZEJ WIŚNIEWSKI
}

(Communicated by Andrew M. Bruckner)

\begin{abstract}
The structure of measurable linear functionals and operators on Fréchet spaces with so-called stochastic bases is described.
\end{abstract}

\section{INTRODUCTION}

In this paper we study measurable linear functionals and operators on Fréchet spaces. Measurable linear functionals have been studied by many authors (see e.g., $[1,4,6,10,11])$, however, measurable linear operators have been seldom treated. The theory of measurable linear operators on Hilbert spaces was presented in [10] and on Banach spaces in [12].

The purpose of this paper is to investigate the structure of measurable linear functionals and operators on Fréchet spaces with so-called stochastic bases. The notion of the stochastic basis was introduced by Herer in [3]. In this paper we will admit a slight modification of this concept.

Our results extend and generalize the corresponding facts that have been proved for particular cases (see $[2,4,8])$. However, all these cases, until now, have been treated separately. In this paper they will appear as the corollaries of our results. Moreover, we shall present a lot of new similar examples.

Our results were announced previously in [13] without proofs.

\section{Preliminaries}

Let $X$ and $Y$ be real separable Fréchet spaces (i.e., complete locally convex linear metric spaces), and let $\mu$ be a Borel probability measure on $X$. Denote by $\mathscr{B}(X)$ the Borel $\sigma$-algebra on $X$ and by $\mathscr{B}_{\mu}(X)$ the completion in measure $\mu$ of $\mathscr{B}(X)$.

An operator $A$ defined on a linear subset $D_{A} \subset X$ with values in $Y$ is called a $\mu$-measurable linear operator if

(a) $D_{A} \in \mathscr{B}_{\mu}(X)$ and $\mu\left(D_{A}\right)=1$;

(b) $A$ is a measurable mapping with respect to $\left(\mathscr{B}_{\mu}(X), \mathscr{B}(Y)\right)$;

(c) $A$ is linear on $D_{A}$.

Received by the editors March 10, 1990.

1991 Mathematics Subject Classification. Primary 60B11, 28C20, 46G12; Secondary 46A06.

Key words and phrases. Measurable linear functionals, measurable linear operators, Fréchet space, Gaussian measure, stable measure, product measure. 
If $Y=R$ then we will say simply functional instead of operator.

Denote by $X^{*}$ the topological dual of $X$, and let $\left\{x_{n}, f_{n}\right\}$ be a biorthogonal system in $\left(X, X^{*}\right)$, i.e., $f_{i}\left(x_{j}\right)=\delta_{i j}$ for $i, j=1,2, \ldots$. Such a system is called a stochastic basis in $(X, \mu)$ if

(a) there exists a Borel subset $T_{\mu} \subset X$ with $\mu\left(T_{\mu}\right)=1$ such that for every $x \in T_{\mu}$

$$
\lim _{n \rightarrow \infty} \sum_{k=1}^{n} f_{k}(x) x_{k}=x ;
$$

(b) functionals $\left\{f_{n}\right\}$ treated as random variables on $(X, \mu)$ are stochastically independent.

Therefore, if $\left\{x_{n}, f_{n}\right\}$ is a stochastic basis in $(X, \mu)$ then we can write that

$$
x=\sum_{n=1}^{\infty} f_{n}(x) x_{n}
$$

for every $x \in T_{\mu}$.

It is evident that $T_{\mu}$ is a linear subset of $X$.

The measure $\mu$ will be called nondegenerate if the measure of every proper closed linear subspace in $X$ is less than 1 .

We will say that the measure $\mu$ is symmetric if $\mu(B)=\mu(-B)$ for every $B \in$ $\mathscr{B}(X)$. By $\mu^{s}$ we shall denote the symmetrization of $\mu$, that is, a symmetric measure defined by the formula $\mu^{s}(B)=\frac{1}{2}(\mu(B)+\mu(-B))$ for $B \in \mathscr{B}(X)$.

\section{Main Results}

Let $X$ be a separable Fréchet space, and let $\mu$ be a Borel probability measure on $X$. Suppose now that there exists a stochastic basis $\left\{x_{n}, f_{n}\right\}$ in $(X, \mu)$.

Proposition 1. Assume that the measure $\mu$ is nondegenerate. If $L$ is a $\mu$ measurable linear subset of $X$ such that $\mu(L)=1$ then $x_{n} \in L$ for every $n=1,2, \ldots$.

Proof. Suppose in the first place that the measure $\mu$ is symmetric. Denote by $S$ the set of linear transformations $s: T_{\mu} \rightarrow T_{\mu}$ such that $s\left(x_{n}\right)=\varepsilon_{n} x_{n}$ where $\varepsilon_{n}= \pm 1$ and $\varepsilon_{n}=1$ for all $n \geq n_{0}$ for some $n_{0} \geq 1$. Therefore if $x \in T_{\mu}$ and $x=\sum_{n} f_{n}(x) x_{n}$ then $s(x)=\sum_{n} \varepsilon_{n} f_{n}(x) x_{n} . S$ is obviously a countable set. Since the measure $\mu$ is symmetric and functionals $\left\{f_{n}\right\}$ are independent as random variables on $(X, \mu)$, we have that

$$
\mu(s(B))=\mu(B)
$$

for all $B \in \mathscr{B}(X),\left(B \subset T_{\mu}\right)$, and every $s \in S$.

Since $\mu$ is a Radon measure on $X$, we can find a $\sigma$-compact linear set $L_{0} \subset L$ such that $\mu\left(L_{0}\right)=\mu(L)=1$. So we may as well assume that $L$ itself is a $\sigma$-compact and consequently a Borel subset of $X$.

Let $L_{1}=L \cap T_{\mu}$. Then $L_{1}$ is a linear Borel set and $\mu\left(L_{1}\right)=1$. Let us put

$$
L_{0}=\bigcap_{s \in S} s\left(L_{1}\right) .
$$

$L_{0}$ is a linear Borel set and from (1) it follows that $\mu\left(L_{0}\right)=1$. 
Now let $n \geq 1$ be fixed. Then there is $y \in L_{0}$ such that $f_{n}(y) \neq 0$. In fact, if for every $y \in L_{0}, f_{n}(y)=0$, then $\mu\left\{y \in X: f_{n}(y)=0\right\}=1$, which is impossible because the measure $\mu$ is nondegenerate.

Let $s \in S$ be the transformation with $\varepsilon_{n}=-1$ and $\varepsilon_{k}=1$ for $k \neq n$. Since $s\left(L_{0}\right)=L_{0}$ then $s(y) \in L_{0}$. Let us remark that $y-s(y)=2 f_{n}(y) x_{n}$. Hence $x_{n}=\left(2 f_{n}(y)\right)^{-1}(y-s(y))$. Moreover, since $L_{0}$ is a linear set and $y$, $s(y) \in L_{0}$, we have $x_{n} \in L_{0}$. But $L_{0} \subset L_{1}$ whence $x_{n} \in L_{1}$ and consequently $x_{n} \in L$. This proves our fact in the case when $\mu$ is a symmetric measure.

If now $\mu$ is any measure then we may consider the symmetrization $\mu^{s}$ of $\mu$. Since $T_{\mu}$ is a linear set and $\mu\left(T_{\mu}\right)=1, \mu^{s}\left(T_{\mu}\right)=1$. Therefore $\left\{x_{n}, f_{n}\right\}$ is also a stochastic basis for $\mu^{s}$. Moreover, since $\mu$ is a nondegenerate measure, so is $\mu^{s}$. Thus if $L$ is a $\mu$-measurable linear set such that $\mu(L)=1$ then also $\mu^{s}(L)=1$ and by virtue of the first part of this proof, we have that $x_{n} \in L$ for every $n=1,2, \ldots$. This completes the proof of our assertion.

Now let

$$
C(\mu)=\left\{\left(a_{n}\right) \in R^{\infty}: \sum_{n=1}^{\infty} a_{n} f_{n}(x)<\infty \mu \text {-almost everywhere }\right\} .
$$

It is evident that if $\left(a_{n}\right) \in C(\mu)$ then the formula

$$
f(x)=\sum_{n=1}^{\infty} a_{n} f_{n}(x)
$$

defines a $\mu$-measurable linear functional on $X$. Our aim will be to show that for the symmetric and nondegenerate measure $\mu$, every $\mu$-measurable linear functional on $X$ has a representation in the form (2).

Let us before remark that if $f$ is a $\mu$-measurable linear functional defined on $D_{f}$ and if we suppose that the measure $\mu$ is nondegenerate, then by Proposition 1 we have $x_{n} \in D_{f}$ for all $n=1,2, \ldots$. Therefore every $\mu$-measurable linear functional is in this case defined for each $x_{n}(n=1,2, \ldots)$. The after-mentioned fact shows that the values of the functional $f$ on vectors $x_{n}$ $(n=1,2, \ldots)$ uniquely determine this functional.

Proposition 2. Suppose that the measure $\mu$ is nondegenerated, and let $f$ be a $\mu$-measurable linear functional on $X$ (defined on $D_{f}$ ). If $f\left(x_{n}\right)=0$ for $n=1,2, \ldots$ then $f=0$-a.e.

Proof. For every $x \in D_{f} \cap T_{\mu}$ and for all $n=1,2 \ldots$, we have

$$
x=\sum_{k=1}^{n} f_{k}(x) x_{k}+\sum_{k=n+1}^{\infty} f_{k}(x) x_{k} .
$$

Hence

$$
f(x)=\sum_{k=1}^{n} f_{k}(x) f\left(x_{k}\right)+f\left(\sum_{k=n+1}^{\infty} f_{k}(x) x_{k}\right)=f\left(\sum_{k=n+1}^{\infty} f_{k}(x) x_{k}\right) .
$$

Thus $f$ depends only on the random variables $\left\{f_{k}: k \geq n+1\right\}$ for every $n=1,2, \ldots$ But the random variables $\left\{f_{n}\right\}$ are independent on $(X, \mu)$. Hence by virtue of the Kolmogorov's $0-1$ law, we obtain that there is $c \in R$ 
such that $f=c \mu$-a.e. Since the functional $f$ is linear then $c=0$. Therefore $f=0 \mu$-a.e.

Corollary. Suppose that the measure $\mu$ is nondegenerate. If $f$ and $g$ are $\mu$ measurable linear functionals on $X$ and $f\left(x_{n}\right)=g\left(x_{n}\right)$ for $n=1,2, \ldots$, then $f=g \quad \mu$-a.e.

The main results of this paper are the following two theorems.

Theorem 1. Assume that the measure $\mu$ is symmetric and nondegenerate. If $f$ is a $\mu$-measurable linear functional on $X$ then

(a) $\left\{f\left(x_{n}\right)\right\}_{n=1}^{\infty} \in C(\mu)$;

(b) $f(x)=\sum_{n=1}^{\infty} f_{n}(x) f\left(x_{n}\right) \quad$ u-a.e.;

(c) the representation (b) is unique.

Proof. (a) Let $x \in D_{f} \cap T_{\mu}$ and $n \in N$. Then

$$
x=\sum_{k=1}^{n} f_{k}(x) x_{k}+x(n), \quad \text { where } x(n)=\sum_{k=n+1}^{\infty} f_{k}(x) x_{k} .
$$

Hence

$$
f(x)=\sum_{k=1}^{n} f_{k}(x) f\left(x_{k}\right)+f(x(n)) .
$$

Define $a_{n}=f\left(x_{n}\right), X_{n}(x)=a_{n} f_{n}(x)$, and $Y_{n}(x)=f(x(n))$. Then $X_{1}, X_{2}, \ldots$ is a sequence of independent and symmetric random variables on $(X, \mu)$. Also for every $n \in N$ the random variables $X_{1}, X_{2}, \ldots, Y_{n}$ are independent.

The equality (3) can be rewritten in the form

$$
f(x)=\sum_{k=1}^{n} X_{k}(x)+Y_{n}(x) .
$$

Denote by $\varphi, \varphi_{n}$, and $\psi_{n}$ the characteristic functions of $f, X_{n}$, and $Y_{n}$. Then from (4) we have

$$
\varphi(t)=\psi_{n}(t) \prod_{k=1}^{n} \varphi_{k}(t)
$$

Hence

$$
|\varphi(t)| \leq \lim _{n \rightarrow \infty} \prod_{k=1}^{n}\left|\varphi_{k}(t)\right| .
$$

Since $X_{1}, X_{2}, \ldots$ are independent random variables, the series $\sum_{n=1}^{\infty} X_{n}$ is either convergent $\mu$-a.e. or divergent $\mu$-a.e. If this series is divergent then by [ 7 , Corollary 2, p. 251, Theorem b, p. 250] we have that $\lim _{n \rightarrow \infty} \prod_{k=1}^{n}\left|\varphi_{k}(t)\right|=0$. Then from (5) it follows that $\varphi(t)=0$ for $t \neq 0$. But this is impossible because $\varphi$ is a characteristic function (of the random variable $f$ ). Therefore $\sum_{n=1}^{\infty} X_{n}<\infty \mu$-a.e., that is, $\sum_{n=1}^{\infty} f\left(x_{n}\right) f_{n}(x)<\infty \mu$-a.e. This means that $\left\{f\left(x_{n}\right)\right\}_{n=1}^{\infty} \in C(\mu)$.

(b) Let $g(x)=\sum_{n=1}^{\infty} f_{n}(x) f\left(x_{n}\right)$. Since by (a) $\left\{f\left(x_{n}\right)\right\} \in C(\mu), g$ is a $\mu$-measurable linear functional on $X$. But $f\left(x_{n}\right)=g\left(x_{n}\right)$ for every $n \in$ 
$N$. Hence by the Corollary we have that $f=g \mu$-a.e., that is, $f(x)=$ $\sum_{n} f_{n}(x) f\left(x_{n}\right) \quad \mu$-a.e.

(c) Suppose that the functional $f$ has another representation in the form (b), i.e., let $f(x)=\sum_{n} a_{n} f_{n}(x) \mu$-a.e. But then $f\left(x_{n}\right)=a_{n}$ for $n=1,2, \ldots$. Thus the representation (b) is really unique.

Theorem 2. Let the measure $\mu$ be symmetric and nondegenerate, and let $Y$ be a separable Banach space. If $A$ is a $\mu$-measurable linear operator from $X$ into $Y$ then $A$ may be uniquely represented in the form

$$
A x=\sum_{n=1}^{\infty} f_{n}(x) A x_{n} \quad \text { M-a.e. }
$$

Proof. For every $f \in Y^{*}$ the formula $f_{A}(x)=f(A x)$ defines a $\mu$-measurable linear functional on $X$. Therefore by Theorem 1 we obtain

$$
f_{A}(x)=\sum_{n=1}^{\infty} f_{n}(x) f_{A}\left(x_{n}\right) \quad \mu \text {-a.e. }
$$

or

$$
f(A x)=\sum_{n=1}^{\infty} f_{n}(x) f\left(A x_{n}\right) \quad \mu \text {-a.e. }
$$

for every $f \in Y^{*}$.

Regarding $A$ as a random vector with values in $Y$, we have $\left\{f_{n}(x) A x_{n}\right\}_{n=1}^{\infty}$ is a sequence of independent and symmetric random vectors and by Theorem Ito-Nisio [5] we obtain (6). The uniqueness of (6) also follows from Theorem 1.

\section{EXAMPLES}

Now we will present some examples of measures for which there exist stochastic bases and consequently Theorems 1 and 2 about the structure of measurable linear functionals and operators hold. In any case we also want to give some information about the set $C(\mu)$, that characterizes the space of $\mu$-measurable linear functionals.

Example 1. Let $X$ be a separable Fréchet space and $\mu$ a symmetric Gaussian measure on $X$. Then there is a stochastic basis in $(X, \mu)$ (see [3]). It is easy to show that in this case $C(\mu)=l_{2}$.

For the particular case of the Gaussian measure on a separable Banach space Theorem 1 has been obtained in [8].

Example 2. Let $X$ be a separable Banach space and $\mu$ a symmetric $p$-stable measure with discrete spectral measure $(0<p<2)$. This case was considered in [2] where the construction of a stochastic basis for such measure $\mu$ was given. It was also shown that in this case $C(\mu)=l_{p}$.

Example 3. Let $X=R^{\infty}$ and $\mu=\prod_{n=1}^{\infty} \mu_{n}$ be a probability product measure on $X$ with symmetric and nondegenerate factors. Measurable linear functionals for such measures $\mu$ have been considered in detail in [4]. But it is easy to see that this example is also a special case of our results. Indeed, if $e_{n}$ is the 
$n$th unit vector in $R^{\infty}$ and $f_{n}(x)=x_{n}$ for $x=\left(x_{n}\right) \in R^{\infty}$ then the system $\left\{e_{n}, f_{n}\right\}$ is a stochastic basis in $\left(R^{\infty}, \mu\right)$.

The set $C(\mu)$ depends in this case on measures $\mu_{n}$. If, for example, all measures $\mu_{n}$ are identical then $C(\mu) \subset l_{2}$ (see [4]). For other information about the set $C(\mu)$ in this case we refer to paper [4].

Example 4. Let $X$ be a separable Fréchet space and $\mu$ a symmetric measure of second order on $X$ (i.e., $\int_{X}\|x\|^{2} d \mu(x)<\infty$ for every continuous seminorm \|\| in $X$ ).

Measurable linear functionals and operators for such measures have not been considered before. But in this case Theorems 1 and 2 also hold if we suppose that there exist sufficiently rich stochastically independent continuous linear functions on $X$. Indeed if $\mu$ is a symmetric measure of second order on $X$ and if we suppose that there exists a sequence $\left\{g_{n}\right\} \subset X^{*}$ such that $\left\{g_{n}\right\}$ are stochastically independent random variables on $(X, \mu)$ and that $\left\{g_{n}\right\}$ is total in $X^{*}$ with respect to the $L^{2}(\mu)$-metric, then there exists a stochastic basis $\left\{x_{n}, f_{n}\right\}$ in $(X, \mu)$ (see [9]).

Now we show that in this case $C(\mu) \supset l_{2}$.

Let us remark that without loss of generality (by [9, Theorem 2]) we can assume that $X$ is a separable Banach space (with the norm \|\| ). We may also suppose that $\left\|f_{n}\right\|=1$ for $n=1,2, \ldots$.

To prove that $l_{2} \subset C(\mu)$ let us assume that $\left(a_{n}\right) \in l_{2}$ and put $X_{n}(x)=$ $a_{n} f_{n}(x) \quad(n=1,2, \ldots)$. Then $\left\{X_{n}\right\}$ is a sequence of independent random variables on $(X, \mu)$. Moreover for every $n \in N$ we have

$$
E X_{n}^{2}=\int_{X}\left(a_{n} f_{n}(x)\right)^{2} \mu(d x) \leq a_{n}^{2} \int_{X}\|x\|^{2} \mu(d x) .
$$

Hence

$$
E\left(\sum_{j=n+1}^{n+m} X_{j}^{2}\right) \leq \sum_{j=n+1}^{n+m} E X_{j}^{2} \leq \int_{X}\|x\|^{2} \mu(d x) \cdot \sum_{j=n+1}^{n+m} a_{j}^{2} .
$$

Since $\mu$ is a measure of second order, $\int_{X}\|x\|^{2} \mu(d x)<\infty$. Hence, taking into account the assumption (i.e., that $\left(a_{n}\right) \in l_{2}$ ), we infer from (8) that the series $\sum_{n} X_{n}$ is convergent in $L_{2}(\mu)$ and consequently also in probability $\mu$. But the random variables $\left\{X_{n}\right\}$ are independent, therefore from the convergence of the series $\sum_{n} X_{n}$ in probability $\mu$ follows the convergence of this series $\mu$-almost everywhere (see [5, Theorem 3.1]). Thus $\sum_{n} X_{n}<\infty \mu$-a.e., that is, $\sum_{n} a_{n} f_{n}(x)<\infty \mu$-a.e. This means that $\left(a_{n}\right) \in C(\mu)$. Our statement is proved.

\section{REFERENCES}

1. R. Cameron and R. Graves, Additive functionals on a space of continuous functions. I, Trans. Amer. Math. Soc. 70 (1951), 160-176.

2. Dang Hung Thang and Nguen Zui Tien, On symmetric stable measures with discrete spectral measure on Banach spaces, Lecture Notes in Math., vol. 828, Springer-Verlag, Berlin, Heidelberg, and New York, 1980, pp. 286-301.

3. W. Herer, Stochastic bases in Fréchet spaces, Demonstratio Math. 14 (1981), 719-723.

4. J. Hoffman-Jørgensen, Integrability of seminorms, the 0-1 law and the affine kernel for product measures, Studia Math. 61 (1977), 137-159. 
5. K. Ito and M. Nisio, On the convergence of sums of independent Banach space valued random variables, Osaka Math. J. 5 (1968), 35-48.

6. M. Kanter, Random linear functionals and why we study them, Lecture Notes in Math., vol. 645, Springer-Verlag, Berlin, Heidelberg, and New York, 1978, pp. 114-123.

7. M. Loeve, Probability theory, 3rd ed., van Nostrand, Toronto, New York, and London, 1963.

8. Nguen Zui Tien, The structure of measurable linear functionals for Gaussian measures on Banach spaces, Teor. Veroyatnost. i Primenen. 23 (1978), 165-168. (Russian)

9. Y. Okazaki, Stochastic basis in Fréchet space, Math. Ann. 274 (1986), 379-383.

10. A. V. Skorohod, Integration in Hilbert spaces, Springer-Verlag, Berlin, Heidelberg, and New York, 1974.

11. K. Urbanik, Random linear functionals and random integrals, Colloq. Math. 33 (1975), 255-263.

12. A. Wiśniewski, Measurable linear operators on Banach spaces, Colloq. Math. 54 (1987), 261-265.

13. __ Measurable linear functionals and operators on Fréchet spaces, Fifth Internat. Vilnius Conf. on Probab. Theory Math. Stat., Abstracts of Comm., T.II, Vilnius, 1989, pp. 227-228.

Institute of Mathematics, SzcZecin University, Ul. Wielkopolska 15, 70-451 SzcZecin, Poland

Current address (to July 1992): Department of Mathematik, ETH-Zentrum, Rämistrasse 101, CH-8092 Zürich, Switzerland 